\title{
USABLE, REAL-TIME, INTERACTIVE SPOKEN LANGUAGE SYSTEMS
}

\author{
John Makhoul and Madeleine Bates, Principal Investigators \\ BBN Systems and Technologies \\ 70 Fawcett St. \\ Cambridge, MA 02138
}

\begin{abstract}
PROJECT GOALS
The primary objective of this project is develop a robust, high-performance, domain-independent spoken language system. The system, termed HARC (Hear And Respond to Continuous speech), is composed of the BYBLOS speech recognition and the DELPHI natural language understanding system. The goal is to develop systems that exhibit the following advances: high-accuracy speech understanding with vocabulary of up to 10,000 words; a highly interactive user interface capable of mixed-initiative dialogue and other types of system feedback; transparent adaptability to new users; easy portability to new applications; a system implementable in real-time on cost-effective COTS (commercial, off-the-shelf) hardware.
\end{abstract}

\section{RECENT RESULTS}

- Developed the first complete real-time spoken language system on a workstation, without the use of additional hardware. The system integrated our real-time speech recognition and NL systems through the $\mathrm{N}$-best interface. The real-time system was demonstrated in the ATIS domain at the DARPA 1992 and 1993 workshops on speech and natural language processing. The system was developed on a Silicon Graphics Indigo workstation and has been ported to other Unix-based workstations.

- Built a new semantic interpretation component that enables a cleaner separation between the domainindependent knowledge stored in a general grammar of English and the domain-dependent knowledge associated with particular lexical items, thus enhancing portability and domain-independence. The new component is based on the notion of translating from grammatical relations to semantic relations.

- Developed a hybrid approach to semantic representation that combines the expressive power of logic with the reasoning power of frames. This allows the system to detect contradictions (e.g., "ffights to denver ... uhh to Boston") without being forced into the simple frame-slot approach that is inadequate for many types of natural language phenomena.
- Developed the Semantic Linker, a new fallback understanding component using probabilities that reduces the understanding error rate by $30 \%$. The Semantic Linker makes extensive use of the hybrid representation and interpretation component above to take over when DELPHI's regular parser cannot understand an input utterance.

- Laid out an initial design and developed an initial representation for a new statistical model of understanding. the Hidden Undersatanding Model. The new model will enable us to carry out studies in the alignment of corpora to semantic interpretations, with the goal of automatically deriving semantic rules.

- Participated in the November 1992 speech, NL and SLS evaluations, and in the end-to-end log-file evaluation dry-run experiment.

- Chaired the organization of the March 1993 DARPA Human Language Technology workshop.

\section{PLANS FOR THE COMING YEAR}

Our plans for the coming year center on extending the work above in several areas. In particular, we plan to:

- Integrate the Semantic Linker and the parser, to increase the capabilities of both.

- Improve our discourse understanding component, making it more domain-independent.

- Develop ways of extending our grammar for different domains. This will allow us to keep our domainindependent knowledge base, but to tailor it for domainspecific syntactic constructions.

- Continue the development of the Hidden Understanding Model and test it by deriving the semantic rules for a real domain.

- Collect additional ATIS data for the general community, using our real-time system and the new, expanded database. 